\title{
Germanica
}

\section{„Arno Schmidt ist ein Sonderfall“. Monitum einer Kritik Marcel Reich-Ranickis}

André KISCHEL : "Arno Schmidt ist ein Sonderfall ».Critique d'une recension de Marcel Reich-Ranicki

André KISCHEL: "Arno Schmidt ist ein Sonderfall". Critique of a Review by

Marcel Reich-Ranicki

\section{André Kischel}

\section{OpenEdition \\ Journals}

Édition électronique

URL : https://journals.openedition.org/germanica/7566

DOI : 10.4000/germanica.7566

ISSN : 2107-0784

\section{Éditeur}

Université de Lille

\section{Édition imprimée}

Date de publication : 1 décembre 2019

Pagination : 47-64

ISBN : 978-2-913857-44-5

ISSN : 0984-2632

Référence électronique

André Kischel, ${ }_{n}$ Arno Schmidt ist ein Sonderfall“. Monitum einer Kritik Marcel Reich-Ranickis“,

Germanica [Online], 65 | 2019, Online erschienen am: 01 Januar 2022, abgerufen am 08 Januar 2022.

URL: http://journals.openedition.org/germanica/7566 ; DOI: https://doi.org/10.4000/germanica.7566 


\title{
„Arno Schmidt ist ein Sonderfall“" Monitum einer Kritik Marcel Reich- Ranickis
}

\author{
André Kischel \\ Universität Rostock
}

Sie könnten unterschiedlicher kaum sein. Als Antipoden im bundesdeutschen Literaturbetrieb stehen Marcel Reich-Ranicki und Arno Schmidt, wenn auch nicht einander gegenüber, so doch weitest möglich voneinander entfernt. Sie trennt dabei nicht nur ihre Stellung in bzw. zu diesem Betrieb; der eine Kritiker im Zentrum, der andere Autor, zumindest in der allgemeinen Wahrnehmung, eher am Rande. Hatte der eine von Berufs wegen mit dem anderen zu tun, so mied der andere den einen, eben wegen dessen Beruf.

Und doch gibt es Schnittmengen zwischen diesen beiden Persönlichkeiten. Beide sind kritische Beobachter ihrer Zeit, finden in ihrem Schreiben deutliche Worte, fällen harte, bisweilen schroffe Urteile über andere, nicht selten an der Grenze zur Beleidigung. Und beide betonen auf die eine oder andere Weise den eigenen exzeptionellen Status. Etwa indem sie versuchen, einen eigenen literarischen Kanon zu stiften und durchzusetzen. Nicht nur durch die Auswahl der von ihm besprochenen Autoren, sondern auch ganz offensiv mit seinen Anthologie- und Kanon-Reihen verfolgt Reich-Ranicki dieses Ziel.

1. - Marcel Reich-Ranicki an Peter Rühmkorf, 18.12.2000, in: Christoph Hilse, Stephan Opitz (Hrsg.), Marcel Reich-Ranicki-Peter Rühmkorf. Der Briefwechsel, Göttingen, Wallstein, 2015, S. 243. 
Dabei steht das Gros der von ihm ausgewählten Schriftsteller und Werke kaum zur Diskussion, vielmehr wird in der Regel eine weit gefasste, bereits allgemein konsensfähige Auswahl deutschsprachiger Literatur damit bestätigt. Schmidt hingegen präsentiert einen Privatkanon von teils weitgehend vergessenen, teils durchaus noch tradierten Autoren, denen er mehr Wahrnehmung wünscht. Für sich reklamiert er dabei das Verdienst der Wiederentdeckung.

Vielleicht liegt in dieser Parallele schon ein erster Grund für die unüberbrückbare Distanz zwischen Schmidt und Reich-Ranicki. Der Kritiker schreibt über den Autor, wenigstens ein einziges Mal; der Autor hingegen hält sich grundsätzlich mit öffentlichen Reaktionen auf Kritik bedeckt. Dieses Verhältnis im Nicht-Verhalten zueinander soll hier näher beleuchtet werden. Da Reich-Ranickis Name in keiner Publikation Schmidts zu finden ist, liegt der Schwerpunkt im Folgenden zwangsläufig auf Reich-Ranickis Perspektive.

In zwei Schritten wird nachvollzogen, wie der Kritiker den Autor im aktuellen Literaturbetrieb verortet und sodann bewertet. Dabei ist zu beobachten, wie sich Reich-Ranicki zugleich selbst positioniert. Wiewohl er für sich Objektivität beansprucht, greift er Schmidt erheblich an. Oft bleibt dabei jedoch unklar, was er statt des von ihm Kritisierten eigentlich erwartet. In einem dritten Schritt soll es um die Nachwirkungen dieser Kritik gehen. Schmidts Name wird für Reich-Ranicki zu einem pars pro toto für eine ganze Schriftstellergeneration. Abschließend wird die Blickrichtung gewechselt und geprüft, wie Schmidt sich zu Literaturkritik verhalten hat.

\section{Schmidt wird verortet}

„Meine längste Kritik behandelte Arno Schmidt“2, behauptet ReichRanicki 1993 in einem Interview. Ob es sich tatsächlich um seine „längste Kritik“ handelt, sei dahingestellt. Gemeint hat er mit Sicherheit die umfangreiche, am 13. Oktober 1967 in der Zeit veröffentlichte Besprechung Schmidts unter dem Titel ,Selfmadeworld in Halbtrauer“. Um eine Literaturkritik, wie sie das Tagesgeschäft des Feuilletons ist, handelt es sich dabei allerdings nicht. Es gibt keinen, zumindest unmittelbar aus dem Text erkennbaren Anlass für diese Kritik. Sie scheint vielmehr darauf angelegt zu sein, mit einem Rundumschlag das Thema Arno Schmidt einmal ausführlich abzuhandeln, um es dann nicht mehr berühren zu müssen.

Rückblickend sieht Reich-Ranicki in der Tatsache, dass er „ohne aktuellen Anlaß einen überdimensionierten Aufsatz über Arno Schmidt““

2. - „, Kritiker sind einsam“. Marcel Reich-Ranicki über sich selbst und die deutsche Literatur", Der Spiegel, 40/1993, S. 279-287, hier S. 287. 
schreiben konnte, über den sein Redakteur Dieter E. Zimmer zwar „stöhnte“, dann aber „das riesige Manuskript sofort und ungekürzt“" drucken ließ, einen Ausweis für seine unbestrittene Sonderrolle im Feuilleton der $\mathrm{Zeit}^{3}$. Es war also vielleicht schlicht an der Zeit, dass Reich-Ranicki sich wenigstens einmal zu Schmidt äußerte ${ }^{4}$. Das verleiht dieser einen Äußerung um so mehr Gewicht.

„Alljährlich“" lege Schmidt Bücher vor, und wenngleich ReichRanicki bisher keines besprochen hat, möchte er für 1967 konstatieren: „Ein Ärgernis ist Arno Schmidt längst nicht mehr"5. Schmidt legt in diesem Jahr kein eigenes Buch vor, er tritt lediglich als Mit-Übersetzer des zweiten Bandes der Werke Edgar Allen Poes im Walter-Verlag in Erscheinung. Seit Schmidt in den 1950er Jahren als Provokateur debütierte und dieses Image eine Weile habe pflegen können, sei viel Zeit vergangen. Die einst extremen Urteile über ihn, zwischen bedingungsloser Begeisterung und absoluter Ablehnung habe es keine Schattierungen gegeben, seien inzwischen allerdings verklungen; niemand würde heute, 1967, mehr für ihn derart in die Bresche springen, „niemand legt seine Worte auf die Waagschale, niemand will ihm widersprechen" (237). Womit Reich-Ranicki Schmidt vorweg schon sehr unvermittelt relative Bedeutungslosigkeit bescheinigt. Das sei nun aber ein Zustand, mit dem Schmidt sich nicht abfinden könne. Die inzwischen erfolgte Anerkennung durch die literarische Öffentlichkeit widerspreche seinem Selbstbild, ,ein heftig umstrittener Schriftsteller“ zu sein, und Reich-Ranicki folgert, Schmidt sehne ,sich nach jenen Zeiten zurück“ (237). Für den Kritiker ist es nun legitim, wie gleichermaßen in Literaturkritik und Feuilleton häufiger Usus, Aussagen aus Romanen und Erzählungen, mithin fiktionalen Texten, für solche ihres Autors zu nehmen. Eine erzählende und also vermittelnde Instanz, und damit Distanz, die zu berücksichtigen keineswegs nur literaturwissenschaftlich geboten ist, wird schlichtweg übergangen. So will er den Autor Schmidt mit einem Zitat aus Das steinerne Herz charakterisieren, das durchaus geeignet scheint, als eine Leserapostrophe des Erzählers, freilich nicht des Autors, gelten zu können: „Wenn mich die

3. - Marcel Reich-Ranicki, Mein Leben, München, DTV, 2000, S. 466.

4. - Vgl. Karl-Heinz Müther, Bibliographie Arno Schmidt 1914-1979. Grundwerk und Nachlieferungen 1-18, Bielefeld, Aisthesis, 1992-2017. Reich-Ranicki taucht hier sehr wohl mehrfach auf, allerdings in der Regel in seiner Funktion als Herausgeber von Sammelbänden, in denen Texte Schmidts veröffentlicht wurden. Als einzige Besprechung ist nur der hier verhandelte Text zu finden.

5. - Marcel Reich-Ranicki, ,Selfmadeworld in Halbtrauer“, Die Zeit, 13.10.1967, zit. nach: Marcel Reich-Ranicki, Literatur der kleinen Schritte. Deutsche Schriftsteller in den sechziger Jahren, erw. Ausgabe, München, DTV, 1992, S. 236-259, hier S. 236. Im Folgenden wird auf die Seiten dieser Textausgabe mittels Klammern hinter dem Zitat verwiesen. 
Offiziellen loben: dann iss Zeit aufhören!" Reich-Ranicki kommentiert: „Zum Glück steht er nicht zu seinem Wort" $(238)^{6}$. Er begrüßt und befürwortet hier oberflächlich, dass Schmidt weiterschreibt, auch noch als ihm offizielle Anerkennung widerfährt. Allerdings ist diese schon recht implizite, ironische Würdigung in eine Diskreditierung des mit dem Erzähler gleichgesetzten Autors verpackt, der eben sein Wort nicht hält - ein gemeinhin negativ bewertetes Verhalten. Ironie hat stets diese Eigenschaft, ,auf die eine oder andere Weise mit Kritik verbunden“ zu sein 7 . Hier kaschiert das Lob den Tadel kaum, es ist vergiftet.

Trotz solch einleitendem Präjudiz, behauptet Reich-Ranicki für seine Auseinandersetzung, dass Schmidt nicht „mit extremen Urteilen beizukommen" ist (239). Er gibt seiner Besprechung einen Anschein von Objektivität. Denn nicht nur frühe extreme Urteile, sondern auch die - teils von Schmidt selbst wie auch seinen Bewunderern verbreitete - „Aura“ des Autors hätten „die sachliche Beurteilung seiner Prosa offenbar erschwert" (239). Reich-Ranicki will nun scheinbar einen Mittelweg gehen: „Mit Verherrlichung ist es hier nicht getan. Aber auch nicht mit Verwerfung" (239). Das deutet eine differenzierte oder doch ausgewogene Auseinandersetzung zumindest an, mit der er sich von Besprechungen und Bewertungen anderer abzuheben versucht.

Sein Profil des Autors entwickelt Reich-Ranicki dann mit der Beobachtung, dass Schmidts Arbeit von „bemerkenswerter Akribie“ zeuge und eine ,außergewöhnliche Systematik“ aufweise (240). Worin diese genau bestehen oder sich niederschlagen, bleibt dabei zunächst offen. So kann er beispielsweise „ein System [...] in seiner Orthographie nicht feststellen“" (256). Stattdessen werden die genannten Eigenschaften als beinah pathologische Symptome einer autodidaktischen Persönlichkeit verstanden, die ursächlich sei für „eine sonderbare Weltfremdheit“, die letztendlich zu „Übertreibungssucht“, „Einseitigkeit“" (240), und schließlich auch ,ins Provinzelle“ (242) führe. Neben dieser bedenklichen ,Diagnose“ ist in ihrem Umfeld insbesondere

6. - Reich-Ranicki verkürzt hier, das Zitat lautet in der Erstausgabe: „Wenn mich die Offiziellen loben werden: dann iss Zeit aufhören!" (Arno Schmidt, Das steinerne Herz. Historischer Roman aus dem Jahr 1954, Karlsruhe, Stahlberg, 1956, S. 178). Die Aussage zielt so deutlicher auf ein künftiges, womöglich irreales Ereignis. Was Reich-Ranicki nicht wissen konnte: Es handelt sich hier um eine Passage, die auf die Bedenken des Verlegers Ernst Krawehl hin vor der Veröffentlichung, mit Duldung des Autors, politisch entschärft wurde. Die Bargfelder Ausgabe hat den ursprünglichen Text wieder hergestellt: „Wenn mich die CDU loben wird, oder das Amt Blank: dann iss Zeit aufhören!“"(Arno Schmidt, Das steinerne Herz. Historischer Roman aus dem Jahr 1954, in: Arno Schmidt, Bargfelder Ausgabe, Werkgruppe I, Bd. 2. Zürich, Haffmans, 1986, S. 7-163, hier S. 103). Ursprünglich sollten ,die Offiziellen' hier also sehr viel konkreter adressiert werden.

7. - Hannele Kohvakka, Ironie und Text. Zur Ergründung von Ironie auf der Ebene des sprachlichen Textes, Frankfurt a.M., Peter Lang, 1997, S. 22. 
ein sprachlicher Aspekt von Bedeutung. Denn Reich-Ranicki problematisiert zwar die Fragwürdigkeit seiner „Verallgemeinerungen“, meint aber gleichwohl, dass die „Stichworte, die sie uns liefern“, durchaus „brauchbar" wären (240). Mit dem in diesem Zusammenhang erstmals verwendeten Personalpronomen ,uns" macht er sich zum Sprecher seines Publikums. Ein rhetorischer Kniff, der sogleich eine Opposition aufbaut, auf der einen Seite ein gemeinschaftliches ,uns 'bzw. ,wir', dem ein ,er', ein Individuum, gegenübersteht. In seiner Sprecherrolle formuliert der Kritiker so vorgeblich Meinung und Urteil einer Gemeinschaft über den Einzelnen. Wie in vielen anderen seiner Texte, bedient sich Reich-Ranicki hier noch mehrfach dieser Methode.

Für Schmidts ,Arriviertheit' im Literaturbetrieb zählt er zwei der drei literarischen Preise auf, die bis dahin an den Autor vergeben wurden. Auch die Aufnahme in Schriftstellerlexika, in solche der BRD wie der DDR, führt er zu Recht als ein weiteres Argument an. Plakativ bedient dabei der von ihm ausgewählte ostdeutsche Lexikonartikel die Erwartungen des westdeutschen Lesers: Schmidt sei ein „bürgerlich-oppositioneller Schriftsteller, der ... scharf gegen Faschismus, Militarismus und jegliche Art von Religion Stellung nimmt" (237-238). Angesichts des kompletten Lexikoneintrags entsteht hier jedoch der Eindruck, als sollte nicht nur das von einem Lexikon der DDR zu erwartende geboten werden, sondern auch etwas nicht geboten werden. Hinter seiner Auslassung verbirgt sich ein Lob der Schmidt'schen Prosa: Er gilt dort als ein „bürgerlich-oppositioneller Schriftsteller, der in seinen mit unbändiger sprachlicher Experimentierfreude geschriebenen kultur- und gesellschaftskritischen ,Kurzromanen' gegen Faschismus" ${ }^{\text {" etc. Position }}$ beziehe. Ferner hält Reich-Ranicki Besprechungen durch „Professoren der Germanistik“ für ein weiteres Indiz (238). Für ,Professoren der Germanistik', gerade solche, die sich mit Gegenwartsliteratur befassen, gehört es zum Beruf, sich mit zeitgenössischen Schriftstellern auseinanderzusetzen, und dabei ist es durchaus legitim, am literaturkritischen Diskurs teilzunehmen. Dass das in den 1960er Jahren noch weitaus seltener der Fall war als heute, steht außer Frage. Die nur schwer zu überprüfende Behauptung aber, dass „Staatsexamens- und Seminararbeiten, ja auch Dissertationen über Arno Schmidt [...] heute keine Seltenheit mehr" seien (238), muss der Leser glauben. Als Übertreibung im zulässigen Sinne einer Literaturkritik und eines der liebsten rhetorischen Instrumente Reich-Ranickis ist sie für den zeitgenössischen Leser kaum zu erkennen. Die insgesamt sehr sorgfältig geführte Bibliographie Karl-Heinz Müthers bestätigt diese Behauptung jedenfalls nicht. Die

8. — „Schmidt, Arno“, in: Deutsches Schriftstellerlexikon. Von den Anfängen bis zur Gegenwart, durchges. Nachdruck d. zweiten Aufl., Weimar, Volksverlag, 1962, S. 515-516, hier S. 515. 
früheste universitäre Arbeit wird dort zwar bereits auf 1963 datiert, bis 1967 sind es dann aber nur drei weitere Haus- bzw. Magisterarbeiten, Dissertationen folgen erst ab $1969^{9}$.

Die aktuelle Situation auf dem Buchmarkt skizziert Reich-Ranicki rasch, indem er feststellt, dass die ,wichtigeren erzählenden und essayistischen Werke Schmidts“ verfügbar seien, Rundfunksender ihn mit Arbeit ,überhäufen“ (238). Für die Übersetzungen aus dem Englischen, die gerade bis in die späten 1960er Jahre hinein einen erheblichen Teil von Schmidts Arbeit ausmachen, hat Reich-Ranicki kaum mehr als einen Satz übrig. Es sei „recht und billig“ (238), ihn diese machen zu lassen. Bis 1967 waren es immerhin 16 Bücher und Mitarbeit an zwei Bänden der Poe-Ausgabe. Offensichtlich ist es Reich-Ranicki nicht an einer Betrachtung des gesamten Schmidt'schen Schaffens gelegen, es steht der Erzähler mit seinem eigenen literarischen Schreiben im Fokus.

Allerdings fällt es schwer, aus den oft negativ konnotierten aufzählenden Beobachtungen die Maßstäbe der Bewertungen abzuleiten. Denn Reich-Ranicki benennt die Kriterien seiner Wertungen nicht, nicht nur hier, sondern grundsätzlich. Seine „literaturkritischen Grundsätze und Maßstäbe" kann und will er nicht offenlegen, denn die „Begegnung mit einem lebendigen Kunstwerk“ schaffe stets aufs Neue „Regeln und Kriterien, Grundsätze und Maßstäbe“10. Als literaturkritisches Programm leuchtet das einerseits ein, wie es andererseits dem Kritiker jede Freiheit gewährt, womit seine Urteile aber auch der Gefahr einer Beliebigkeit ausgesetzt sind. Ein von Reich-Ranicki eingefordertes Kriterium ist ganz offensichtlich das der Originalität, die unbedingt erwartet wird, dem Schmidt in Reich-Ranickis Augen aber nicht gerecht wird. Charakteristisch für Schmidts Euvre ist, dass für viele seiner Naturschilderungen, wie überhaupt für seine Sprache, der Expressionismus „Pate gestanden hat“ (250). Bereits in den frühen Rezensionen zu seinem Debüt Leviathan wurde von verschiedenen Kritikern festgestellt: ,,so dichteten die Super-Expressionisten“, woraus schon damals gefolgert wurde, Schmidt käme ,literaturgeschichtlich zu spät"11. Reich-Ranickis impliziter Vorwurf lautet wiederum, dass sich diese also längst bekannten Stilmittel ja ,,seither leicht nachahmen“ ließen (251). Dieser Tadel der Epigonalität wird noch ausgebaut: Schmidts

9. - Vgl. Karl-Heinz Müther, Bibliographie Arno Schmidt, a.a.O., S. 1497. Vermutlich wird es mehr universitäre Arbeiten gegeben haben, sie nachträglich noch zu bibliographieren ist kaum möglich. Reich-Ranickis Behauptung scheint dennoch reichlich hoch gegriffen.

10. - Marcel Reich-Ranicki, „Vorbemerkung“, in: Ders., Deutsche Literatur in West und Ost, München, Piper, 1963, S. 11-13, hier S. 13.

11. - O., „Verzweifelte Konsequenz“, Stuttgarter Nachrichten, 29.12.1949, zit. nach: Hans-Michael Bock (Hrsg.), Über Arno Schmidt. Rezensionen vom „Leviathan “ bis zur ,Julia“, Zürich, Haffmans, 1984, S. 10-11, hier S. 10. 
poetologisches Programm weiche „nicht im geringsten von jener Aufgabe ab, die sich auch die Realisten des neunzehnten Jahrhunderts gestellt haben“, nur eben mit „neuen Ausdrucksmitteln“ (246) - die Reich-Ranicki zudem nicht ,neu' genug sind. Er zeichnet hier das Bild eines Autors, der formal nicht über den Expressionismus und thematisch nicht über den Realismus des 19. Jahrhunderts hinausgekommen sei.

Bemerkenswert ist die Argumentation dafür. Er bedient sich dazu einer Reihe von Schmidt-Passagen, unter anderem aus dem Kurzroman Aus dem Leben eines Fauns: „Jeder Schriftsteller sollte die Nessel Wirklichkeit fest anfassen; und uns alles zeigen" (246). An anderen Stellen wiederum betont Reich-Ranicki wiederholt Schmidts Positivismus, er sei „verliebt in Ziffern, Atlanten, Lexika“ und erhoffe sich „,von den exakten Wissenschaften eine geradezu rettende Funktion" (242). Im Überblick skizziert er damit eher das Bild eines Naturalisten, der seine ästhetische Wahrheit einer hässlichen Wirklichkeit gleichrangig neben die absolute Wahrheit der naturwissenschaftlich messbaren Welt stellt, beide in ihren Relationen sieht. Das von Reich-Ranicki zitierte (Ost-) Deutsche Schriftstellerlexikon hat diesen Aspekt immerhin gesehen, wenn es, aus seiner ideologisch vorgeprägten Sichtweise freilich mit Bedauern, Schmidts Romanen eine ,naturalistisch[e] Beobachtung“ der jüngsten Vergangenheit und des Zeitgeschehens attestiert ${ }^{12}$. Und selbst wenn man den Naturalismus als eine Weiterentwicklung oder Reaktion auf den poetisch oder bürgerlich genannten Realismus begreift, so ist doch die programmatische Differenz zwischen beiden Literaturentwürfen evident, wie auch die Tatsache, dass Schmidt Elemente von Naturalismus wie Expressionismus weiterentwickelt und produktiv auf seine Gegenwart anwendet. Und auch damit ist Schmidts Werk keineswegs erschöpfend bezeichnet oder gar literarhistorisch verortet. Reich-Ranicki wirft hier die Begriffe durcheinander. So ist Schmidt zwar sehr wohl an einem realistischen Erzählen, einem nach seinen poetologischen Maßstäben orientierten Darstellen der Welt gelegen; keineswegs produziert er aber damit eine Literatur des Realismus des 19. Jahrhunderts. Überdies widerspricht die Reihe der dystopisch konzipierten Erzähltexte, etwa Schwarze Spiegel und Die Gelehrtenrepublik, die Reich-Ranicki auch nennt, der Vorstellung von einer ,rettenden Funktion durch Naturwissenschaften'; wie übrigens auch die oft recht versteckt auftretenden Elemente des Phantastischen, wie etwa in Brand's Haide. Schmidts vermeintlicher ,Positivismus' ist vielmehr ein Ausgangspunkt, eine Art solide Basis für alles Weitere. Unbestritten finden sich in seinen Werken immer wieder Lobgesänge auf die Verlässlichkeit wissenschaftlicher Erkenntnisse, wie auch ein Zürnen über deren Vernachlässigung bzw. ungenaue Anwendung, so

12. — „Schmidt, Arno“, in: Deutsches Schriftstellerlexikon, a.a.O., S. 516. 
etwa in seinem Aufsatz über die fehlerhaften Mondbilder in der Literatur, beispielsweise bei Goethe oder Gottfried Keller13. Überdies relativiert Schmidt selbst seinen ,Positivismus': „Denn wenn ich Zustände und Denkweisen einer Epoche erfahren will, benütze ich mit nichten die Meßtischblätter der Historiker - auf denen kann ich allenfalls exakt Namen \& Daten abgreifen - sondern ich nehme mir für, sagen wir 1770, den ,Werther' zur Hand [...], sowie noch 1 Dutzend anderer Zeitgenossen [...]: und dann weiß ich Bescheid!"14 Diese Haltung, hier vermittelt durch die Sprecherrolle eines Funkdialogs, deckt sich nachweislich mit der Schmidts. In einem Interview mit Martin Walser bestimmte er die Funktion des Schriftstellers dahingehend, ,daß er ein Bild seiner Zeit geben soll, was der Historiker, der nachfühlend eine vergangene Epoche zu beschreiben versucht, niemals geben kann“, denn ein „Historiker kann nur das Meßtischblatt einer Zeit geben, [...] also Daten abgreifen“. Hingegen müsse der Schriftsteller darüber hinausgehen, und „das Porträt des Denkprozesses eines Menschen jener Zeit mitgeben“, jener Zeit nämlich, von und aus der geschrieben wird, und das „,kann nur der zeitgenössische Schriftsteller"15.

Grundsätzlicher und auch persönlicher wird Reich-Ranickis Kritik, wenn er sich mit Schmidts prosatheoretischen Überlegungen auseinandersetzt. Darin erläutert Schmidt u.a. seine sogenannte Rastertechnik. Sie beruht auf der Annahme, dass das Erzählprinzip des epischen Flusses einer getreuen Abbildung der Wirklichkeit, die er anstrebt, nicht genügen könne, gleichfalls eine Illusion sei. Stattdessen seien von den 1440 Minuten eines Tages „höchstens 50 belangvoll“16, und nur sie erzählerisch relevant, mit all den damit notwendig einhergehenden Zeit- und Ereignisbrüchen. Dass diese Erkenntnis für die Literatur der 1950er und 1960er Jahre nicht unbedingt originell ist, steht außer Frage, Reich-Ranicki betont es. Neben anderen, teils berechtigten Einwänden, die sämtlich auf den vermissten Neuigkeitswert zielen, stellt er hier aber einen bemerkenswerten Schluss an: Ausgehend von Schmidts Prosa und seinen theoretischen Erläuterungen nimmt Reich-Ranicki an, dass sich dahinter eben kein poetologisches Programm verbirgt, sondern Schmidts Literatur von seinen „schriftstellerischen Möglichkeiten“

13. - Vgl. Arno Schmidt, „Finster war's der Mond schien helle“, in: Ders., Bargfelder Ausgabe, Werkgruppe III, Bd. 3, Zürich, Haffmans, 1995, S. 122-123, hier S. 122.

14. - Arno Schmidt, „Der sanfte Unmensch“, in: Ders., Bargfelder Ausgabe, Werkgruppe II, Bd. 2, Zürich, Haffmans, 1990, S. 61-85, hier S. 63.

15. - Rundfunkinterview von Martin Walser für den Süddeutschen Rundfunk, in: Arno Schmidt, Bargfelder Ausgabe, Supplemente, Bd. 2, Frankfurt a.M., Suhrkamp, 2006, S. 9-11, hier S. 9.

16. - Arno Schmidt, „Berechnungen I“, in: Ders., Bargfelder Ausgabe, Werkgruppe III, Bd. 3, a.a.O., S. 163-168, hier S. 167. 
begrenzt werde, es würden überhaupt ,von ihm nur diese Rasterpunkte oder Moleküle wahrgenommen". Schmidt sei deswegen sogar essentiell „auf die Technik der Montage und Collage angewiesen“, weil ihm andere ,Möglichkeiten', sprich Fähigkeiten, eben fehlten (248). Hier geschieht eine völlige Verdrehung der Gegenstände, ohne dass ein Argument abgesehen von unterstellter Unfähigkeit - für diese Sichtweise vorgebracht wird. Denn Reich-Ranickis Folgerungen geschehen, indem er in Schmidts Texten eine Form vorfindet, die der Autor in seinen erzähltheoretischen Überlegungen erläutert und begründet, um daraus den Vorwurf zu konstruieren, dass der Autor eben nur solche Literatur hervorzubringen vermöge. Auf diesen ,Zirkelschluss' folgt die Behauptung, diese „kurzen Sequenzen“ Schmidts seien häufig ,austauschbar“, womit eine Beliebigkeit unterstellt und einem sinnstiftenden Konstruktionsprinzip widersprochen wird. Die dahinterstehende Montage- und Collagetechnik sieht Reich-Ranicki nicht, er verteidigt Schmidt gegenüber ein eher traditionell-konventionelles Erzählprinzip, wie es etwa bei Thomas Mann oder Theodor Storm zu finden ist. Schmidts Texte sind in dieser Lesart nicht mehr als sozusagen willkürlich aneinandergereihte, literarisch bestenfalls passable Prosafragmente, seine Poetologie darüber ,wertlos“ (247).

Zwei Jahre später legt Reich-Ranicki noch einmal nach: In seiner Rezension von Günter Grass' Roman Örtlich betäubt möchte er die geringe erzähltechnische Innovationskraft hervorheben. Als Vergleich wird Arno Schmidt herangezogen, den er als den „konsequentesten Nachfolge[r]" von Alfred Döblins ,Kinostil' ansieht, in dessen erzählerischer Tradition Grass wie Schmidt stünden. Am Beispiel Schmidts sei zu sehen, wie diese Form letztendlich ,zur unfreiwilligen Komik führen"würde"17.

Reich-Ranickis Vorgehen folgt den Spielregeln der Literaturkritik. Einleitend stellt er Autor und Werk vor, wobei er schon allein durch seine Wortwahl deutlich macht, was er von Schmidt hält, nämlich nicht allzu viel. Diese Haltung hebt er sodann ins Allgemeine, indem er sich zum Sprecher des Publikums macht. Dabei reizt er die der Literaturkritik inhärente Subjektivität freizügig für seine Zwecke aus. Die angekündigte Objektivität entpuppt sich als Feigenblatt.

\section{Schmidt wird bewertet}

Um nicht einen totalen Verriss vorzulegen, entdeckt Reich-Ranicki hier und da aber auch Aspekte in Schmidts Werk, die sein Wohlgefallen finden. So verfüge Schmidt über, ,poetische[n] Furor“, der zusammen mit

17. - Marcel Reich-Ranicki, „Eine Müdeheldensoße. Zum zweitenmal: Der neue Roman von Günter Grass“, Die Zeit, 29.8.1969, S. 16. 
seiner ,ungewöhnlichen akustischen Reizbarkeit“ ein geeignetes Mittel sei, „die Sprache der einfachen Leute“ aufzunehmen und festzuhalten (248). Überraschenderweise treffen hier Reich-Ranickis Beobachtungen mit Schmidts schriftstellerischem Anspruch einigermaßen harmonisch aufeinander. Will Schmidt als Schriftsteller die ,Denkprozesse einer Zeit' stenografieren, so lobt Reich-Ranicki, dass es ihm nicht nur gelingt, „Denkschablonen, Verhaltensmuster und klischeehafte Gefühlsreaktionen in den dargestellten Milieus aufzudecken“, sondern ihnen darüber hinaus auch noch „Poesie abzugewinnen“ (249). Dieses Lob wird zwei Jahre später von Reich-Ranicki graduell deutlicher formuliert, wenngleich es an erster Stelle Manfred Bieler gilt, dem Autor des Romans Maria Morzeck oder Das Kaninchen bin ich (1969): Es habe „niemand seit Arno Schmidts ,Steinernem Herzen“ die Ausdrucksweise der Berliner, zumal der kleinen Leute, so genau und treffend belauscht und so komisch wiedergegeben"18. Spricht er bei Schmidt von der ,ungewöhnlichen akustischen Reizbarkeit und Empfänglichkeit', so ist bei Manfred Bieler von einer ,besonderen akustischen Reizbarkeit und Beobachtungsgabe“ die Rede ${ }^{19}$.

Mit einigem Wohlwollen findet Reich-Ranicki in Schmidt nun nicht nur jenen „Mathematiker und Systematiker“, sondern auch noch einen „Idylliker", der jedoch - kein Lob ohne ein Aber - ,an seiner Sentimentalität leidet". Da sich Schmidt, in Reich-Ranickis Lesart, „seiner Zärtlichkeit schämt", sie „verbergen möchte“, würde er solche Textpassagen häufig durch „provozierend geschmacklose Akzente“ brechen (249). Nur warum ein sich seiner Zärtlichkeiten schämender Autor diese überhaupt publiziert, bleibt offen. Außerdem kann die Provokation nun auch so groß wiederum nicht sein, man habe sich doch inzwischen daran gewöhnt. Als Exempel liefert Reich-Ranicki zwei Passagen aus dem Kurzroman Aus dem Leben eines Fauns. Hier stört ihn, dass zwei idyllische Szenen - in der einen pflückt Protagonist Düring Pflaumen mit der von ihm umschwärmten Käthe, in der anderen beobachtet er sie bei der Morgentoilette - von der Mitteilung unterbrochen werden, dass Düring Schweißfüße hat. Reich-Ranickis Wiedergabe verkürzt auf entstellende Weise, denn es gibt Gründe für diesen ,Bruch“. Nach dem Pflaumen-Erlebnis kehrt Düring in sein wenig geliebtes Heim zu seiner ebenso wenig geliebten Ehefrau zurück. Dass hier nun seine stinkenden Füße thematisiert werden, passt da ins Bild. Sodann beginnt

18. - Marcel Reich-Ranicki, „Eine schnoddrige Berliner Romanze. Manfred Bielers Buch Maria Morzeck oder Das Kaninchen bin ich“, Die Zeit, 10.10.1969, S. 21.

19. - Ebd. Wenige Zeilen nach der zitierten Passage folgt auch der beinah unvermeidlich scheinende Verweis auf Döblin. Reich-Ranicki, wie wohl die meisten ,Berufsschreiber', seien es Kritiker oder Schriftsteller oder sonstige, hat eben auch seine Phrasen, Versatzstücke und ,Sequenzen'. Sollten sie ihm vorgeworfen werden, wie er es bei Schmidt macht? 
ein neuer Tag, an dem er endlich eine ersehnte Reise antreten kann, der mit einem „Gut Zeichen“ beginnt ${ }^{20}$, dem Fensterblick auf die sich offenherzig waschende Angebetete, also einer neuen mehr oder weniger, idyllisch-zärtlichen' Szene. Der ,geschmacklose Akzent" wird in Anbetracht des Kontextes sinnfällig, er unterstreicht die Opposition zwischen der ,Zärtlichkeit' mit der Geliebten und dem häuslichen Trott mit der Gattin. Wie schon beim Deutschen Schriftstellerlexikon hilft auch hier eine verkürzte Darstellung der Argumentation des Kritikers.

Die Betrachtung des ,Idyllikers' führt Reich-Ranicki dann auch zu Schmidts Naturschilderungen. Die grundsätzlich expressionistisch inspirierte Konstruktionsweise hat er bereits kritisiert, und wiederholt sich bei dieser Gelegenheit. Wenn er dabei vermeintlich einschränkt, dass diese Bilder nicht ,,ausnahmslos alle [...] fragwürdig oder minderwertig“" seien (252), sagt er damit genau das Gegenteil, die Mehrheit sei es eben doch. Und schließlich findet der Kritiker dann auch endlich die bereits mehrfach postulierte ,Systematik'. Er vermutet, dass „Schmidts Metaphern ganz unabhängig von Schmidts Romanen und Erzählungen entstanden" seien, vielmehr auf Vorrat produziert und dann nach Bedarf und Belieben in seine Texte montiert würden (252). So stört er sich bei Schmidts Sprachbildern an den vielfältigen Desubstantivierungen und besonders an ,Genitivmetaphern', die ,preziös und pathetisch klingen“" (253). Was aber wäre die Alternative zu einer Formulierung wie „die schwarze Pfanne der Nacht" (253)? Doch wohl kaum eine ,schwarze Nachtpfanne', in der das weiter ausgeführte Naturbild dann womöglich einen ,geschmacklosen Akzent' bekäme, wenn der Mond, wie ein „Spiegelei drin gebraten“, illustriert wird als ,[b]laßgelber Dotter, wabbeliges Wolkenweiß" darum ${ }^{21}$. Dem Lyriker Peter Huchel war diese Metapher aus Das steinerne Herz immerhin gut genug für eines seiner Gedichte: „Knisternd fiel die Hitze / Aus glühender Pfanne der Nacht.“22 - Auch für den Lyriker ist nur diese, von Reich-Ranicki Genitivmetapher genannte Form sinnvoll möglich, um das gewünschte Bild zu erzeugen. Indem er schließlich noch Schmidts Darstellungen von Sexualität kurzerhand für „,vollends lächerlich“ (253) erklärt, beendet Reich-Ranicki seine Obduktion am Werk und bereitet sein finales Urteil vor.

Zunächst liefert er noch eine Kollegenschelte, wenigstens aber eine Selbsterhöhung. Er erklärt Schmidts frühe Erfolge, die im Wesentlichen

20. - Arno Schmidt, ,Aus dem Leben eines Fauns“, in: Arno Schmidt, Bargfelder Ausgabe, Werkgruppe I, Bd. 1, Zürich, Haffmans, 1987, S. 299-390, hier S. 350.

21. - Arno Schmidt, Das steinerne Herz, Bargfelder Ausgabe, a.a.O., S. 120.

22. - Peter Huchel, „Polybios I“, in: Axel Vieregg (Hrsg.), Peter Huchel. Gesammelte Werke in zwei Bänden, Bd. 1, Frankfurt a.M., Suhrkamp, 1984, S. 149-150, hier S. 150. Das Gedicht erschien erstmals 1963 in Huchels Gedichtband Chausseen, Chausseen. Eine Verbindung zwischen Huchel und Schmidt soll damit nicht unterstellt sein, der intertextuelle Verweis ist aber deutlich. 
nur in der Literaturkritik, kaum aber auf dem Buchmarkt stattfanden, mit mangelnder Lesekompetenz des betreffenden Publikums. Denn es sei vor allem „die Sprache dieser Prosa“ gewesen, die „einen Teil der jungen Generation über die Schwächen der Epik Schmidts hinwegzutäuschen vermochte" (254). Dafür hat die zuvor noch heftig kritisierte Sprache also genügt, um seinen Kollegen der 1950er Jahre nachträglich Oberflächlichkeit unterstellen zu können.

Sodann verdeutlicht Reich-Ranicki, was unter der ,Provinzialität ${ }^{\star}$ zu verstehen sei, die er in seinem ,Psychogramm' Schmidts festgestellt hat. So seien etwa die Schilderungen Ost-Berlins in Das Steinerne Herz zwar ,eng und bescheiden“ (254), gleichwohl - oder eben dadurch würde er ,zum epischem Chronisten seiner Zeit“, wenn er die „Provinz aus der Perspektive eben dieser Provinz" zeige (255). Damit versucht Reich-Ranicki nicht nur den Horizont der Figuren, sondern auch den ihres Autors abzustecken. Ob es dann allerdings das Anliegen Schmidts gewesen ist, mit Die Gelehrtenrepublik, ,den Horizont seiner Epik entschieden erweitern" (255) zu wollen, wird eine Spekulation des Kritikers bleiben müssen. Die Protagonisten in Schmidts Romanen ähneln in ihrer Mentalität einander, Reich-Ranicki hat es in der Sache richtig festgestellt, die Erzähltechnik ähnelt sich ebenfalls, die erzählten Orte und Ereignisse unterscheiden sich, grundsätzlich aber ,geschieht sehr wenig" in Schmidts Romanen (246). In Die Gelehrtenrepublik wird die Dystopie einer Welt nach einem dritten Weltkrieg skizziert, in der die menschlichen und politischen Spielregeln, die zu diesem Krieg führten, nach wie vor Bestand haben. In der Erzähltechnik greift er hier auf sein bereits im Steinernen Herz verwendetes Instrumentarium zurück, was Reich-Ranicki ihm vorwirft. Warum Die Gelehrtenrepublik aber der Versuch sein sollte, weniger, provinziell' zu sein, als eben beispielsweise Das steinerne Herz, lässt Reich-Ranicki offen. Man kann nur vermuten, dass es ihm an dieser Stelle um eine Art geopolitischer Perspektive geht. Allerdings besieht sowohl Protagonist Winer in der Gelehrtenrepublik Folgen und Fortsetzung des „Ost-West-Konflikts“ (255), wie sich auch Eggers im Steinernen Herz seines Ortes, in einem Zentrum eben dieses Konflikts, durchaus bewusst ist, wenn er in dem 1956 publizierten Roman beispielsweise schon fast prophetisch vermutet, dass irgendwann sich „die Ostzone ja aus reinem Selbsterhaltungstrieb abriegeln“ werde müssen ${ }^{23}$. Damit blieben nur die erzählten Orte als sozusagen Prüfsteine der ,Provinzialität', womit aber kaum gedeckt ist, was Reich-Ranicki damit bezeichnen will. Mit einem solchen Blick wären auch das Davos des Zauberbergs, Kafkas Schloss oder auch die Stationen von Hermann Hesses Siddhartha irgendwie recht ,provinziell‘. Der hier leere Begriff ist schlicht ungeeignet für die versuchte Charakterisierung von Schmidts

23. - Arno Schmidt, „Das steinerne Herz“, Bargfelder Ausgabe, a.a.O., S. 161. 
Literatur, er funktioniert hingegen verlässlich für dessen Aburteilung. Gute 30 Jahre später wird Reich-Ranicki, von Peter Rühmkorf auf dieses Thema angesprochen, um Nachsicht bitten, er sei ,eben ein unverbesserlicher Asphaltliterat" 24 . Schmidts Literatur, in aller Regel ländlich situiert, kann dem ,Asphalt‘ wenig abgewinnen und hatte wohl schon deshalb einen schlechten Stand bei Reich-Ranicki.

Mit seinem Urteil über KAFF auch Mare Crisium scheint ReichRanicki dann endgültig, und ohne auch nur einen Anschein von Objektivität beibehalten zu wollen, die Grenze zur Polemik zu überschreiten. Er referiert sehr kurz die Eckpunkte des Romans, stellte dabei fest, dass „Schmidts Darstellungsmethode“ sich hier ,ad absurdum“ führe und konstatiert, der Roman sei ,schlechthin unlesbar“ (256). Dass es sich um eine Zuspitzung handelt, ein bewährtes Mittel der Kritik, steht außer Zweifel. Doch in der hier vorgelegten Weise führt sie sich selbst ,ad absurdum', wenn der Kritiker erst verschiedene Aspekte des Textes moniert, und ihn dann für „unlesbar“ erklärt ${ }^{25}$. Trotz aller Oberflächlichkeit und bissiger Kritik macht Reich-Ranicki dennoch eine aus heutiger Sicht zutreffende Beobachtung, wenngleich sie nicht so gemeint sein konnte und als weiterer Makel daherkommt. Er zieht mit Blick auf $K A F F$ und den Prosaband Kühe in Halbtrauer das Resümee, Schmidt habe „offenbar das Ende jener Sackgasse erreicht, in der er sich schon seit geraumer Zeit befand“ (257). In der gegenwärtigen Schmidt-Forschung gilt $K A F F$ in der Tat, je nach Lesart, als Endpunkt des Frühwerks bzw. als Gelenkstück zwischen Früh- und Spätwerk. Schmidts nächstes Buch wird sein Opus magnum Zettel's Traum sein. Insofern hat Schmidt hier in der Tat das Ende einer literarischen Schaffensphase erreicht, keineswegs ist es aber eine Sackgasse ${ }^{26}$. Da jedoch sieht ihn Reich-Ranicki und so gestaltet sich auch sein Fazit zum Schriftsteller Arno Schmidt. Dieser habe zwar zu einer bestimmten Zeit einen gewissen Effekt und damit verbundene Aufmerksamkeit

24. - Marcel Reich-Ranicki an Peter Rühmkorf, 18.12.2000, in: Christoph Hilse, Stephan Opitz (Hrsg.), Marcel Reich-Ranicki-Peter Rühmkorf, a.a.O., S. 243. In diesem Kontext fällt auch die Aussage, Schmidt sei ein „Sonderfall“, denn es sei bei ihm „,vollkommen gleichgültig, ob die Sache in Berlin stattfindet oder in einem einsamen Dorfe im Oldenburgischen" (ebd.). Bemerkenswert ist die unkritische Verwendung des Begriffs durch Reich-Ranicki, musste er doch um dessen politische Vorbelastung wissen - oder nutzte er ihn gerade deswegen? Für Joseph Goebbels war „Asphaltliteratur“ ein geeignetes Schlagwort für unerwünschte Literatur.

25. - Seit 2004 gibt es eine Hörbuchfassung, und derzeit ist die 1994 aufgelegte Fischer-Taschenbuchausgabe des, unleserlichen` Romans noch in der vierten Auflage lieferbar.

26. - Noch 1975, als Reich-Ranicki knapp auf, ,Schmidts monströses Alterswerk ,Abendstunde mit Goldrand“ [sic!]“ hinweist, sieht er ihn in dieser „Sackgasse“ (Marcel Reich-Ranicki, ,Rückkehr zur schönen Literatur. Eine Bilanz aus Anlaß der Frankfurter Buchmesse“, Frankfurter Allgemeine Zeitung, 8.10.1975, S. 21). 
erzielen können, an der Qualität seiner Literatur habe das allerdings kaum gelegen. Lediglich habe er kurz „den Nerv der Zeit treffen und eine starke Wirkung ausüben“ können, sei dann aber „um so schneller veraltet" (258). Da er diesen Effekt nicht dauerhaft erzielen konnte, habe er sich in seine eigene ,selfmadeworld‘ zurückgezogen, in eine „kleinbürgerliche Idylle" (259), und sei in einer Art dauerhaften ,Protestgeste“ gegen die Welt erstarrt.

Als Ergebnis lässt sich festhalten: Reich-Ranicki lässt sich auf Arno Schmidt nicht ein, seine Lektüre bleibt an der Oberfläche. Zur Betrachtung eines Gesamtwerks, die er hier versucht, ist das bis zu einem gewissen Grad zulässig und auch notwendig. Für ein fundiertes Urteil, das hier ebenfalls versucht wird, reicht das allerdings nicht. Gründlich gelesen hat er Schmidts Werk nicht, allenfalls eine kursorisch-fragmentarische Lektüre lässt sich erkennen. So setzt sich ReichRanicki dem Vorwurf aus, den er Schmidt gemacht hat: Die Details und Versatzstücke, die er wahrnimmt, genügen nicht, um ein Bild des Ganzen zu zeigen.

\section{Nachwirkungen: Referenzpunkt Schmidt}

Damit ist für Reich-Ranicki das Thema Schmidt im Wesentlichen erledigt. Dennoch taucht sein Name gelegentlich in anderen Kritiken auf. Hier und da verweist der Kritiker auf den Autor, in der Regel dann, wenn es darum geht, eine bestimmte Art zu schreiben oder eine Phase der deutschen Literatur zu charakterisieren. Schmidt wird Inventar. Als Reich-Ranicki 1967 seinen Schmidt-Essay abliefert, erscheint auch sein Buch Literatur der kleinen Schritte, in dem größere kritische und essayistische Arbeiten zu deutschen Autoren der 1960er Jahre versammelt werden. Die Schmidt-Besprechung ist als umfangreichster Text darin enthalten. Im Nachwort sortiert Reich-Ranicki Schmidt in die erste Generation deutscher Nachkriegsschriftsteller, neben etwa Alfred Andersch, Wolfgang Koeppen oder Heinrich Böll. Ihnen allen sei gemeinsam, dass sie in ihren Figuren, ,vor allem das leidende Opfer der Geschichte" zeigten, ihre fragenden Blicke in die Vergangenheit richteten ${ }^{27}$. Für diese, erste Generation` sei aber gleichermaßen kenn-

27. - Marcel Reich-Ranicki, ,Statt eines Nachworts. Die deutschen Schriftsteller und die deutsche Wirklichkeit", in: Ders., Literatur der kleinen Schritte. Deutsche Schriftsteller in den sechziger Jahren, erw. Neuausgabe, München, DTV, 1991, S. 307320, hier S. 313. In ähnlicher Weise wird Schmidt neben anderen von Reich-Ranicki aufgezählt in den folgenden Besprechungen: Marcel Reich-Ranicki, „Man muß auch was zu sagen haben. Zu Hans Erich Nossacks siebzigstem Geburtstag“, Die Zeit, 29.1.1971, S. 20; Ders., „Anmerkungen zur deutschen Literatur der siebziger Jahre“, in: Ders., Entgegnung. Zur deutschen Literatur der siebziger Jahre, 4. Aufl., München, DTV, 2002, S. 17-35, hier S. 34; Ders.: „Des Mörders betörender Duft. Patrick Süskinds 
zeichnend, dass sie eine Fülle von utopischen Texten hervorgebracht hätten, zu der dann auch KAFF und Die Gelehrtenrepublik gehören. Für ähnliche Kontexte zitiert Reich-Ranicki Schmidt gelegentlich heran, sowohl vor als auch nach seiner langen Besprechung ${ }^{28}$. Auf eine fortgesetzte Beschäftigung kann daraus allerdings nicht geschlossen werden, vielmehr wird auf Schmidt bei Bedarf zurückgegriffen. Dabei bleibt der Kritiker seinem Urteil mit wenigen Variationen treu. Nur beim Thema Sexualität ist er offenbar unentschlossen. So wird Schmidt, neben Koeppen, 1964 in der Zeit von Reich-Ranicki als ein Vorreiter dieses literarischen Themas angesehen, gerade angesichts der Schwierigkeiten, die andere zeitgenössische Autoren damit hätten ${ }^{29}$. Eine ähnliche Pionierrolle wird Schmidt in einer Besprechung von Rolf Dieter Brinkmanns Roman Keiner weiß mehr (1968) zugestanden ${ }^{30}$. Ein halbes Jahr davor galt sein Umgang damit noch als ,lächerlich“ (253).

Abgesehen von solchen eher beiläufigen Erwähnungen wird ReichRanicki in seiner Rolle als literaturkritische Autorität wiederholt auf Schmidt angesprochen. Zwischen 2003 und 2013 konnten Leser der Frankfurter Allgemeinen Zeitung dem Kritiker Fragen stellen, sieben Mal wird er direkt nach Schmidt gefragt, zwei weitere Male erwähnt er ihn in Aufzählungen. Als sich 2004 mehrere Rezensenten mit Schmidt befassen, wird er gefragt, warum er das nicht auch mache. Zwei Jahre zuvor hatte der Suhrkamp Verlag die Rechte an Schmidts Werk vom Verlag Haffmans übernommen und damit begonnen, sowohl die Bargfelder Ausgabe als auch Einzelausgaben herauszugeben. Dadurch gerät Schmidt wieder vermehrt ins Blickfeld der Feuilletons, zudem ist es das Jahr seines 90. Geburtstags. Reich-Ranicki will sich der Schmidt,Mode“ nicht anschließen, er sei schlicht „,kein glühender Enthusiast seiner Prosa“, wiewohl er „in Grenzen sein Talent“" schätze. Er betont, dass Schmidts Werk ,mit extremen Urteilen“ nicht beizukommen, es mit „Verherrlichung“ ebenso wenig getan sei wie mit „Verwerfung“. Diese Formulierungen stammen aus seinem Schmidt-Essay, auf den er dann die fragende Leserin auch hinweist, um „Näheres über Arno

erstaunlicher Roman ,Das Parfum““, Frankfurter Allgemeine Zeitung, 2.3.1985, Bilder und Zeiten, S. 5.

28. - Vgl. etwa: Marcel Reich-Ranicki, „Der Zeuge Koeppen“, in: Ders., Deutsche Literatur in West und Ost, a.a.O., S. 34-54, hier S. 40-41; Ders., „Nossack der nüchterne Visionär. Zum Tode eines großen Repräsentanten der deutschen Prosa nach 1945“, Frankfurter Allgemeine Zeitung, 10.11.1977, S. 23.

29. - Vgl. Marcel Reich-Ranicki, „Sexus und die Literatur“, in: Ders., Literarisches Leben in Deutschland, München, Piper 1965, S. 242-246, hier besonders: S. 244. Der Text erschien ursprünglich unter dem Titel Hüben und drüben. Sexus und die Literatur in der Zeit vom 13. 11.1964.

30. - Vgl. Marcel Reich-Ranicki, „Außerordentlich (und) obszön. Rolf Dieter Brinkmanns Sex-Roman Keiner weiß mehr“, Die Zeit, 26.4.1968, S. 24. 
Schmidts Prosa“" zu erfahren; als ob seit 1967 nichts mehr zu ergänzen gewesen wäre ${ }^{31}$. Ab 1970 beginnt mit Zettel's Traum, gefolgt von Die Schule der Atheisten (1972) und Abend mit Goldrand (1975), erst seine heute als Spätwerk betrachtete Schaffensphase.

Als Reich-Ranicki einige Jahre später explizit gefragt wird: „Was halten Sie von Arno Schmidt? Frühwerk? Spätwerk?“, weicht er aus, verweist auf die von ihm stets gelobte Erzählung Die Umsiedler und erklärt Zettel's Traum für "häufig zu hoch bewertet"32. Er hat also das späte Werk Schmidts durchaus zur Kenntnis genommen, Zettel's Traum habe er gelesen, es allerdings ,unzweifelhaft langweilig“ gefunden ${ }^{33}$. Auf Schmidts Mammutwerk angesprochen, wiederholt er erneut, teils wortgetreu, seine Ansichten von 1967 und gesteht schließlich hinsichtlich des Schmidt'schen Euvres ein, er ,habe keine Lust“, sich „,mit diesen Büchern zu befassen“34. Ein anderer Leser will es dann aber genau wissen und fragt Reich-Ranicki, warum er denn mit Schmidts Werk so wenig anfangen könne. Der Kritiker zeigt sich in einer abwehrenden Reaktion darüber „verwundert“, er habe sich schließlich 1967 doch einmal ausführlich zu dem Autor geäußert. Und immerhin erinnere er sich an zwei Erzählungen „oft und gern“: Die Umsiedler und Seelandschaft mit Pocahontas ${ }^{35}$. Diese beiden von ihm immer wieder genannten Erzählungen haben es dann auch in seinen Kanon der deutschen Erzählliteratur geschafft ${ }^{36}$.

Um die Frage zu beantworten, ob Schmidt denn nun ein „Scharlatan“ oder „ein großer Schriftsteller“ sei, muss Reich-Ranicki weiter ausholen. Er gibt biographische Hinweise, verweist auf Schmidts ,Liebe“ zu „Ziffern, Atlanten, Lexika“, seine Hoffnung auf eine „rettende Funktion“ der Wissenschaft, womit auch sein „Positivismus“ zusammenhänge, und wie sich damit eine gewisse ,Weltfremdheit bemerkbar“ mache. Auch dass der Expressionismus für Schmidts Metaphorik „Pate gestanden" habe, wird nicht vergessen. Reich-Ranicki erklärt sogleich, dass darin eben auch die Schwäche des Schriftstellers Schmidt liege. Denn mit seinen extravaganten Stilmitteln habe er kurzfristig, in den 1950er Jahren, „den Nerv der Zeit treffen und eine starke Wirkung ausüben" können. Schon wenige Jahre später wirke die Wiederholung bzw. Variation dieser Methode allerdings nur ,noch kurios und weltfremd". So habe der Autor nur vorübergehend Relevanz gehabt, dann aber nicht mehr mit den Zeitläuften mithalten können, und in der

31. — „Fragen Sie Reich-Ranicki“, FAZ, 15.2.2004, S. 28.

32. - "Fragen Sie Reich-Ranicki“", FAZ, 25.11.2012, S. 46.

33. - "Fragen Sie Reich-Ranicki“, FAZ, 23.9.2012, S. 25.

34. - „Fragen Sie Reich-Ranicki“", FAZ, 18.4.2010, S. 26.

35. — „Fragen Sie Reich-Ranicki“, FAZ, 6.5.2007, S. 28.

36. - Vgl. Marcel Reich-Ranicki (Hrsg.): Der Kanon. Die deutsche Literatur. Erzählungen, Bd. 8: Joseph Roth bis Arno Schmidt, Frankfurt a.M., Insel 2003, S. 492-576. 
Folge nur noch ,gegen die gesellschaftliche Misere die kleinbürgerliche Idylle" ausgespielt ${ }^{37}$. Die Urteile klingen vertraut. Und in der Tat handelt es sich bei der Antwort auf die im Jahre 2011 gestellte Frage nach Arno Schmidt um ein Kondensat aus dem mittlerweile 44 Jahre alten Schmidt-Essay Reich-Ranickis. Sätze und komplette Absätze finden sich hier wieder abgedruckt, die Aktualisierungen, die zu erwarten gewesen wären, tendieren inhaltlich wie auch bis in die Wortebene gegen Null. Die mangelnde ,Lust ${ }^{\star}$ sich mit dem Autor zu befassen, ist dieser Wiederverwertung eingeschrieben. Dem Bargfelder Boten, jenem Schmidt gewidmeten Periodikum, ist das nicht entgangen. Dort wird in Fleißarbeit verzeichnet, was an aktuellen Äußerungen über ihren Gegenstand, Materialien zum Werk Arno Schmidts, so alles publiziert wird. Diese Bibliographie liefert als Hilfestellung knappe Erläuterungen und erhellende Hinweise über die rein bibliographischen Daten hinaus. Reich-Ranickis Zweitverwertung wird lakonisch kommentiert: „Alter Unverstand in neuen Schläuchen“38.

\section{Perspektivwechsel: Schmidts Schweigen}

Wie eingangs angedeutet, enthält sich Schmidt weitestgehend Äußerungen oder gar Entgegnungen zu Literaturkritik. In einem Brief an den Kritiker Helmut Heißenbüttel erklärt er, dass er sich ,ein Prinzip daraus gemacht" habe, ,nie einem Rezensenten zu antworten“39. Dass er Kritik sehr wohl wahrgenommen hat, davon zeugen seine Briefwechsel, wie auch die Tagebücher seiner Frau Alice. So bedankt er sich schon mal „für die Zusendung“ einer „Rezension: ich habe sie stolz gelesen, und recht dabei was auf mich gehalten! "40. Als der seinerzeit prominente Literaturkritiker Friedrich Sieburg Schmidts Fouqué-Biographie in der Frankfurter Allgemeinen Zeitung bespricht und sich dabei auch grundsätzlich kritisch zu ihrem Autor äußert, erklärt der Getroffene, Sieburg sei „ein großer Lump“, für den Gründer und langjährigen Feuilletonleiter der Zeitung Karl Korn findet er noch deutlichere Worte ${ }^{41}$. All das waren allerdings private Meinungsäußerungen, für eine Veröffentlichung in absehbarer Zeit kaum gedacht. Sie oblagen

37. — „Fragen Sie Reich-Ranicki“, FAZ, 24.4.2011, S. 26.

38. - In letzter Zeit ist erschienen und zu unserer Kenntnis gelangt, Bargfelder Bote, Lfg. 343, 2011, S. 30-32, hier S. 32.

39. - Arno Schmidt an Helmut Heißenbüttel, 6.11.1957, in: Arno Schmidt, Der Briefwechsel mit Alfred Andersch, Bargfelder Ausgabe, Briefe, Bd. 1. Zürich, Haffmans, 1985, S. 136-137, hier S. 136.

40. - Arno Schmidt an Ernst Kreuder, 27.12.1953, in: Arno Schmidt, Briefwechsel mit Kollegen, Bargfelder Ausgabe, Briefe, Bd. 5, Frankfurt a.M., Suhrkamp, 2007, S. 76.

41. - Arno Schmidt an Alfred Andersch, 29.12.1958, in: Arno Schmidt, Der Briefwechsel mit Alfred Andersch, a.a.O., S. 199-201, hier S. 200. 
ursprünglich der Vertraulichkeit, die jeder Korrespondenz zusteht. Heute erhellen sie glücklicherweise das Gesamtbild. Denn in seinen publizierten Texten gibt sich Schmidt deutlich moderater, wenngleich die Tendenz übereinstimmt. So beschäftigt er sich in seinem Artikel Der Dichter und die Kritik, mit den oft negativen, teils drastischen Folgen, die Rezensionen für Schriftsteller haben können. Wenig überraschend steht er auf der Seite des Schriftstellers, dem es nach einem „Zeichen der Anerkennung seiner grausamen, ,selbst=mörderischen" Arbeit" verlangt ${ }^{42}$. Seine Empfehlung lautet daher, am besten überhaupt keine Kritiken zu lesen, sie auch nicht vermittelt zur Kenntnis zu nehmen, denn ,die Kritik liest in 8 Tagen kein Mensch mehr; das Buch steht nach 100 Jahren noch in der Bibliothek" ${ }^{\text {"43 }}$. Und auch wenn Schmidt sich selbst nicht an seine Empfehlung hält, so geht er doch einer Konfrontation mit Literaturkritikern aus dem Weg.

Es sind dann gelegentlich seine Leser oder Unterstützer, die sich für ihn einsetzen. Auf Reich-Ranickis Text folgen teils korrigierende, teils wütende Leserbriefe. Alle von der Zeit abgedruckten Briefe beziehen dabei Position für Schmidt, gegen Reich-Ranicki44.

Schließlich findet sich der Name Reich-Ranicki dann doch einmal bei Arno Schmidt, und zwar in einem Brief an seinen Verleger Ernst Krawehl. 1969 erschien James Joyces Dubliner in der Neuübersetzung von Dieter E. Zimmer, dem Zeit-Redakteur. Krawehl fragte nun, ob Schmidt diese Übersetzung nicht besprechen wolle. Die Antwort fällt deutlich aus: „Die ,Zeit'=Clique - (Reich=Ranicki, Zimmer, Leonhardt) - soll sich selber, gegenseitig, besprechen, ich rühre da, weder pro noch contra, zunächst keinen Finger"45. Und tatsächlich hat es einmal einen direkten Kontakt zwischen Kritiker und Autor gegeben. Wenige Monate bevor sein Essay erscheint, wendet sich Reich-Ranicki im Juni 1967 an Schmidt mit der Bitte um einen Beitrag für eine Festschrift zu Ehren Heinrich Bölls: Arno Schmidt antwortet nicht ${ }^{46}$.

42. - Arno Schmidt, ,Der Dichter und die Kritik“, in: Ders., Bargfelder Ausgabe, Werkgruppe III, Bd. 3, Zürich, Haffmans 1995, S. 390-392, hier S. 392.

43. - Ebd.

44. - Vgl. „War sein Blick ein wenig getrübt?“, Die Zeit, 27.10.1967, S. 46. Der Bargfelder Bote druckt 30 Jahre später einen Leserbrief des jungen Gerd Haffmans' ab, den Die Zeit damals nicht brachte, in dem er Reich-Ranickis Kritik ausführlich und mit stetem Rückgriff auf Schmidts Werk widerspricht und etliche Fehler richtigstellt (vgl. Gerd Haffmanns, „Deutsche Literaturkritik in Ganztrauer: Zu Marcel ReichRanicki: ,Selfmadeworld in Halbtrauer. Das Werk Arno Schmidts“", Bargfelder Bote, Lfg. 214-216, 1997, S. 29-33).

45. - Arno Schmidt an Ernst Krawehl, 30.7.1969, in: Susanne Fischer, Bernd Rauschenbach (Hrsg.), ,Und nun auf, zum Postauto!" Briefe von Arno Schmidt, Berlin, Suhrkamp, 2013, S. 238-239, hier S. 239.

46. - Vgl. den Kommentar zum Brief Heinrich Bölls an Arno Schmidt, 19.2.1957, in: Arno Schmidt, Briefwechsel mit Kollegen, a.a.O., S. 28-29, hier S. 29. 